\title{
Hopf Bifurcation and Control of Magnetic Bearing System with Uncertain Parameter
}

\author{
Jing Wang, ${ }^{1}$ Shaojuan Ma $\mathbb{D}^{1,2,3}{ }^{1}$ Peng Hao, ${ }^{3}$ and Hehui Yuan ${ }^{3}$ \\ ${ }^{1}$ School of Mathematics and Information Science, North Minzu University, YinChuan 750021, China \\ ${ }^{2}$ The Key Laboratory of Intelligent Information and Big Data Processing of NingXia Province, YinChuan 750021, China \\ ${ }^{3}$ Baota Industry Limited Liability Company, YinChuan 750021, China
}

Correspondence should be addressed to Shaojuan Ma; sjma@nmu.edu.cn

Received 25 May 2019; Revised 25 September 2019; Accepted 22 October 2019; Published 5 December 2019

Academic Editor: Lucia Valentina Gambuzza

Copyright (C) 2019 Jing Wang et al. This is an open access article distributed under the Creative Commons Attribution License, which permits unrestricted use, distribution, and reproduction in any medium, provided the original work is properly cited.

In this paper, the Hopf bifurcation and control of the magnetic bearing system under an uncertain parameter are investigated. Firstly, the two-degree-of-freedom magnetic bearing system model with uncertain parameter is established. The method of orthogonal polynomial approximation is used to obtain the equivalent magnetic bearing model which is deterministic. Secondly, combining mathematical analysis tools and numerical simulations, the Hopf bifurcation of the equivalent model is analyzed. Finally, a hybrid feedback control method (linear feedback control method combined with nonlinear stochastic feedback control method) is introduced to control the Hopf bifurcation behavior of the magnetic bearing system.

\section{Introduction}

One of the most innovative developments involves the use of magnetic bearings. They are now being used widely in rotating machinery because of their advantages, including very low friction, no wear, and high rotor speed. The application of magnetic bearing technology has experienced substantial growth since the First International Symposium on Magnetic Bearings was held in 1988. Nowadays, the magnetic bearings play an important role during the aerospace, mechanical engineering, and transportation, and on the base of these applications [1], various problems on magnetic bearing have attracted more and more researchers.

Many achievements in the field of the nonlinear analysis of the magnetic bearing systems were reported [2-4]. The functional principle of the magnetic bearing is shown in Figure 1. Most of the components are of nonlinear characteristics; therefore, the entire system becomes inherently nonlinear. Some results focused on the modeling of nonlinear characteristics $[5,6]$. Nayfeh and Balachandran added a quadratic nonlinear term to the magnetic bearing model [5]. Ji and Hansen [6] considered the cubic nonlinear term into the magnetic bearing system. In addition, owing to the magnetic bearings having the sophisticated nonlinear characteristics, there are many interesting nonlinear phenomena in systems as given in [7], such as jumping, bifurcation, multisolution, sensitivity to initial conditions, and even chaos. References $[8,9]$ utilized numerical simulation methods to analyze the bifurcation phenomena in active magnetic bearing systems. Orbit plot, the bifurcation diagram, the power spectrum, and Poincaré mapping were used in [8] to identify the main factors affecting the dynamic characteristics of an AMB system. Asymptotic perturbation method [10] and the multiple scales method [11] were used to study the dynamical response of the magnetic bearing system with time-varying stiffness. Nonlinear phenomena like period doubling, quasiperiodic motion, and chaos in the presence of geometrical coupling were observed in [12] with numerical simulation. Zhe et al. studied nonlinear dynamic characteristic analysis of the magnetic bearing system based on the cell mapping method [13].

It is important to note that, in most previous works, many authors paid attention to controlling the problems of the magnetic bearing system. Reference [14] introduced a well-developed position controller and different compensation methods to achieve a good running behavior. Robust 


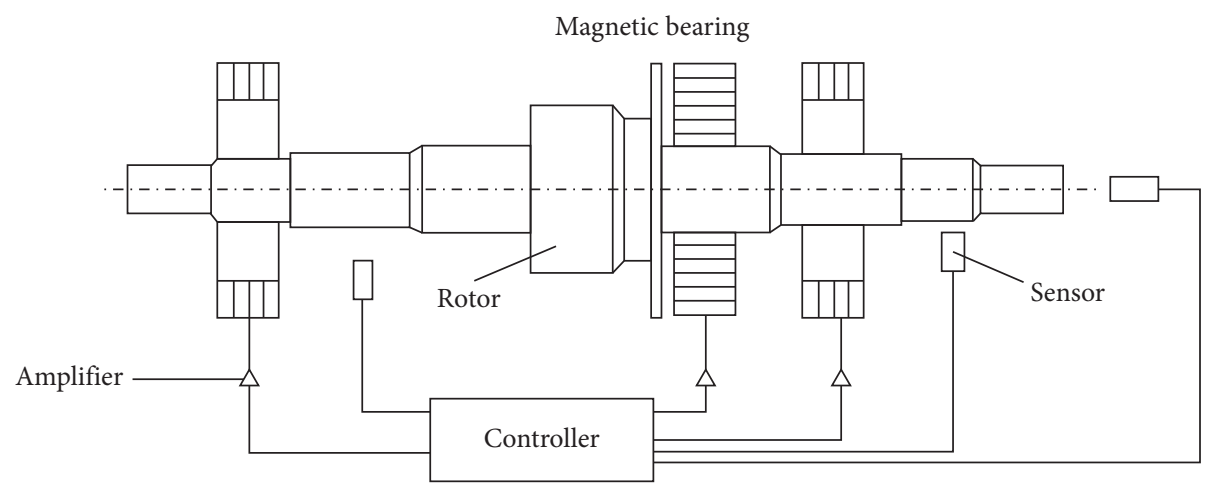

Figure 1: Functional principle of magnetic bearing.

controllers were used to control the effects of external disturbances on magnetic bearings in [15]. Kiani et al. [16] designed a segmented linear form of hybrid controller to stabilize the magnetic bearing system. Shrivastava and his team proposed a model-based method to estimate unbalanced rotor plane parameters, using Kalman filter and recursive least squares input force estimation technique in [17]. The method based on the Fourier series and the Legendre polynomial to identify the imbalance of the magnetic bearing rotor system was described in literature [18]. Reference [19] developed a feedforward control strategy for the rotor imbalance of high-speed magnetic suspension centrifugal compressors.

The above studies on the dynamic behavior and control of bearing are all about deterministic systems. As we all know, uncertainty is ubiquitous. There are some research works which study the dynamic behavior of magnetic bearing systems in random environments. The Monte-Carlo method is used to investigate the bifurcation and chaos characteristics of a cracked rotor with a white noise process as its random disturbance in [20]. Yan and Jia [21] used direct integration scheme in the analysis of the rotor bearing systems subjected to random earthquake excitation. In addition to external random excitation, the randomness of the magnetic bearing itself caused by the environment and materials will also have a certain impact on the system, but such research results have not been found in the literature as far as we know. In this paper, we present stochastic magnetic bearing with uncertain parameters and analyze Hopf bifurcation in detail.

This paper is organized as follows. In Section 2, the stochastic magnetic bearing model is established. Then, we calculate the response solutions of the system. Section 3 develops the Hopf bifurcation of the model and verifies by numerical simulation. The strategies of controlling bifurcation are presented in Section 4.

\section{System Model}

The magnetic bearing is shown in Figure 2. The stator has eight pole pairs. The flux leakage, eddy current loss, saturation, and hysteresis of the core material are ignored as to simplify the model. It is assumed that all magnets have the same structure and number of windings. According to

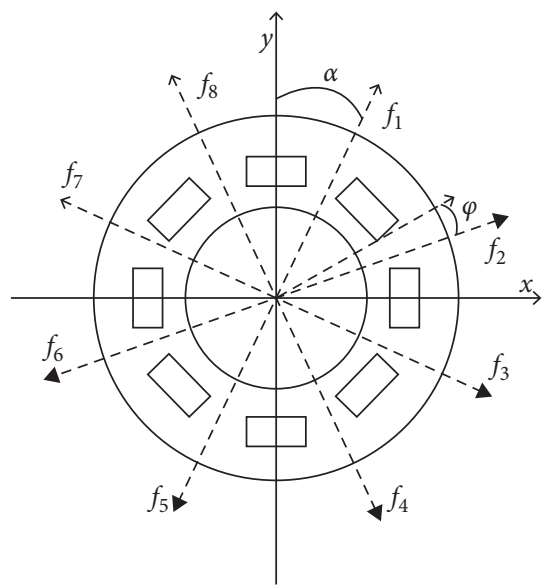

FIgURE 2: Schematic for modeling magnetic forces acting on the bearing.

electric magnetic theory, the magnetic force generated by every pole in the electromagnet can be expressed as follows:

$$
f_{i}=\frac{1}{4} \mu N^{2} A \frac{I_{i}^{2}}{\delta_{i}^{2}} \cos \varphi, \quad i=1,2, \ldots, 8 .
$$

The radial bearing between the electromagnet and the rotor is exemplified by the first pole pair, and the other pole pairs are analyzed in the same way; $x$ and $y$ denote the deviation from the center of the bearing. The radial clearance of the first pole can be written as

$$
\delta_{1}=c_{0}+x \sin \alpha-y \cos \alpha .
$$

The sum electromagnetic forces of the horizontal and vertical directions are of the form as follows:

$$
\begin{aligned}
& f_{x}=\left(f_{2}+f_{3}-f_{6}-f_{7}\right) \cos \alpha+\left(f_{1}+f_{4}-f_{5}-f_{8}\right) \sin \alpha, \\
& f_{y}=\left(f_{4}+f_{5}-f_{1}-f_{8}\right) \cos \alpha+\left(f_{3}+f_{6}-f_{2}-f_{7}\right) \sin \alpha .
\end{aligned}
$$

The force, $f_{i}$, is expanded using a Taylor series and is approximated by only retaining the third-order nonlinear terms; thus, equation (3) can be given by

$$
\begin{aligned}
& f_{x}=f_{x}(\text { linear })+f_{x}(\text { cubic })+o(4), \\
& f_{y}=f_{y}(\text { linear })+f_{y}(\text { cubic })+o(4),
\end{aligned}
$$


where $o(4)$ denotes terms of order greater than four. The model consists of a mass with two degrees of freedom, and the equations of motion governing the unbalance of the magnetic bearing can be written as

$$
\begin{aligned}
& m \ddot{x}=f_{x}-c \dot{x}+m e \Omega^{2} \cos \Omega t, \\
& m \ddot{y}=f_{y}-c \dot{y}+m e \Omega^{2} \sin \Omega t .
\end{aligned}
$$

Finally, introducing nondimensional parameters, and neglecting the rotor weight, the two-degree-of-freedom magnetic bearing system can be rearranged as [6]

$$
\begin{aligned}
& \ddot{x}+2 \mu \dot{x}+\omega^{2} x-\left(\alpha_{1} x^{3}+\alpha_{2} x y^{2}+\alpha_{3} x^{2} \dot{x}+\alpha_{4} \dot{x} y^{2}\right. \\
& \left.\quad+\alpha_{5} x \dot{y}^{2}+\alpha_{6} x \dot{x}^{2}+\alpha_{7} x y \dot{y}\right)=2 f \cos \Omega t, \\
& \ddot{y}+2 \mu \dot{y}+\omega^{2} y-\left(\alpha_{1} y^{3}+\alpha_{2} y x^{2}+\alpha_{3} y^{2} \dot{y}\right. \\
& \left.\quad+\alpha_{4} \dot{y} x^{2}+\alpha_{5} y \dot{x}^{2}+\alpha_{6} y \dot{y}^{2}+\alpha_{7} y x \dot{x}\right)=2 f \sin \Omega t,
\end{aligned}
$$

where $x, y, \mu$, and $\Omega$ refer to the rotor displacement in the $x$ and $y$ directions, magnetic permeability, and rotor speed, respectively. $f$ represents magnetic force, $w$ is the linearized natural frequency, and $\alpha_{1}, \alpha_{2}, \alpha_{3}, \alpha_{4}, \alpha_{5}, \alpha_{6}$, and $\alpha_{7}$ are the coefficients of nonlinear terms. Owing to that the bearing capacity is affected by the medium of the magnetic bearing and it is described by the permeability, we consider $\mu$ is an uncertain parameter. That is to say, its value is not a fixed number, but varies in a small range with magnetic field intensity, temperature and so on. Supposing $\mu$ can be expressed as

$$
\mu=\bar{\mu}+\delta u,
$$

where $\bar{\mu}$ and $\delta / 2$ are the mean and standard deviation of $\mu$, respectively. $u$ is a random variable that defines the probability density function of the arched distribution on $[-1,1]$. Substituting equation (7) into (6), we can get

$$
\begin{aligned}
& \ddot{x}+2(\bar{\mu}+\delta u) \dot{x}+\omega^{2} x-\left(\alpha_{1} x^{3}+\alpha_{2} x y^{2}+\alpha_{3} x^{2} \dot{x}\right. \\
& \left.+\alpha_{4} \dot{x} y^{2}+\alpha_{5} x \dot{y}^{2}+\alpha_{6} x \dot{x}^{2}+\alpha_{7} x y \dot{y}\right)=2 f \cos \Omega t, \\
& \ddot{y}+2(\bar{\mu}+\delta u) \dot{y}+\omega^{2} y-\left(\alpha_{1} y^{3}+\alpha_{2} y x^{2}+\alpha_{3} y^{2} \dot{y}\right. \\
& \left.+\alpha_{4} \dot{y} x^{2}+\alpha_{5} y \dot{x}^{2}+\alpha_{6} y \dot{y}^{2}+\alpha_{7} y x \dot{x}\right)=2 f \sin \Omega t .
\end{aligned}
$$

Equation (8) can be reorganized a first-order standard equation as

$$
\begin{aligned}
\dot{x}= & z, \\
\dot{z}= & 2 f \cos \Omega t+\alpha_{4} y^{2} z-2(\bar{\mu}+\delta u) z+\alpha_{3} x^{2} z+\alpha_{6} x z^{2} \\
& +\alpha_{1} x^{3}+\left(\alpha_{2} y^{2}+\alpha_{5} q^{2}+\alpha_{7} y q-\omega^{2}\right) x, \\
\dot{y}= & q, \\
\dot{q}= & 2 f \sin \Omega t+\alpha_{4} x^{2} q-2(\bar{\mu}+\delta u) q+\alpha_{3} y^{2} q+\alpha_{6} y q^{2} \\
& +\alpha_{1} y^{3}+\left(\alpha_{2} x^{2}+\alpha_{5} z^{2}+\alpha_{7} x z-\omega^{2}\right) y .
\end{aligned}
$$

Equation (9) can be transformed to an equivalent deterministic system through the orthogonal polynomial approximation theory as [22]

$$
\begin{aligned}
\frac{\mathrm{d}}{\mathrm{d} t} x_{0}(t)= & z_{0}(t), \\
\frac{\mathrm{d}}{\mathrm{d} t} z_{0}(t)= & 2 f \cos \Omega t+\alpha_{4} S_{0}(t)-2 \bar{\mu} z_{0}(t) \\
& -\delta z_{1}(t)+\alpha_{3} A_{0}(t)+\alpha_{6} B_{0}(t)+\alpha_{1} X_{0}(t) \\
& +\alpha_{2} C_{0}(t)+\alpha_{5} D_{0}(t)+\alpha_{7} E_{0}(t)-\omega^{2} x_{0}(t), \\
\frac{\mathrm{d}}{\mathrm{d} t} y_{0}(t)= & q_{0}(t), \\
\frac{\mathrm{d}}{\mathrm{d} t} q_{0}(t)= & 2 f \sin \Omega t+\alpha_{4} F_{0}(t)-2 \bar{\mu} q_{0}(t) \\
& -\delta q_{1}(t)+\alpha_{3} G_{0}(t)+\alpha_{6} H_{0}(t)+\alpha_{1} Y_{0}(t) \\
& +\alpha_{2} I_{0}(t)+\alpha_{5} J_{0}(t)+\alpha_{7} K_{0}(t)-\omega^{2} y_{0}(t),
\end{aligned}
$$$$
\frac{\mathrm{d}}{\mathrm{d} t} x_{1}(t)=z_{1}(t),
$$$$
\frac{\mathrm{d}}{\mathrm{d} t} z_{1}(t)=\alpha_{4} S_{1}(t)-2 \bar{\mu} z_{1}(t)-\delta\left(z_{0}(t)+z_{2}(t)\right)
$$$$
+\alpha_{3} A_{1}(t)+\alpha_{6} B_{1}(t)+\alpha_{1} X_{1}(t)+\alpha_{2} C_{1}(t)
$$$$
+\alpha_{5} D_{1}(t)+\alpha_{7} E_{1}(t)-\omega^{2} x_{1}(t)
$$

$$
\begin{aligned}
\frac{\mathrm{d}}{\mathrm{d} t} x_{N}(t)= & z_{N}(t) \\
\frac{\mathrm{d}}{\mathrm{d} t} z_{N}(t)= & \alpha_{4} S_{N}(t)-2 \bar{\mu} z_{N}(t)-\delta z_{N-1}(t)+\alpha_{3} A_{N}(t) \\
& +\alpha_{6} B_{N}(t)+\alpha_{1} X_{N}(t)+\alpha_{2} C_{N}(t)+\alpha_{5} D_{N}(t) \\
& +\alpha_{7} E_{N}(t)-\omega^{2} x_{N}(t)
\end{aligned}
$$

$$
\begin{aligned}
\frac{\mathrm{d}}{\mathrm{d} t} y_{N}(t)= & q_{N}(t) \\
\frac{\mathrm{d}}{\mathrm{d} t} q_{N}(t)= & \alpha_{4} F_{N}(t)-2 \bar{\mu} q_{N}(t)-\delta q_{N-1}(t)+\alpha_{3} G_{N}(t) \\
& +\alpha_{6} H_{N}(t)+\alpha_{1} Y_{N}(t)+\alpha_{2} I_{N}(t)+\alpha_{5} J_{N}(t) \\
& +\alpha_{7} K_{N}(t)-\omega^{2} y_{N}(t) .
\end{aligned}
$$

We make $N=2$, the numerical solution $x_{i}(t), z_{i}(t)$, $y_{i}(t)$, and $q_{i}(t)(i=0,1,2)$ of equation (10) can be obtained by an effective numerical method. The approximate random response of equation (10) can be expressed as 


$$
\begin{aligned}
& x(t, u)=\sum_{i=0}^{2} x_{i}(t) U_{i}(u), \\
& z(t, u)=\sum_{i=0}^{2} z_{i}(t) U_{i}(u), \\
& y(t, u)=\sum_{i=0}^{2} y_{i}(t) U_{i}(u), \\
& q(t, u)=\sum_{i=0}^{2} q_{i}(t) U_{i}(u) .
\end{aligned}
$$

If the random parameter $u=0$, that is to say, when $\mu=\bar{\mu}$, the sample response of the mean parameter system (for short SRM) can be calculated as

$$
\begin{aligned}
& x(t, 0)=\sum_{i=0}^{2} x_{i}(t) U_{i}(0)=x_{0}(t)-x_{2}(t), \\
& z(t, 0)=\sum_{i=0}^{2} z_{i}(t) U_{i}(0)=z_{0}(t)-z_{2}(t), \\
& y(t, 0)=\sum_{i=0}^{2} y_{i}(t) U_{i}(0)=y_{0}(t)-y_{2}(t), \\
& q(t, 0)=\sum_{i=0}^{2} q_{i}(t) U_{i}(0)=q_{0}(t)-q_{2}(t) .
\end{aligned}
$$

The ensemble mean response (for short EMR) of equation (10) is

$$
\begin{aligned}
& E[x(t, u)]=\sum_{i=0}^{2} x_{i}(t) E\left[U_{i}(u)\right]=x_{0}, \\
& E[z(t, u)]=\sum_{i=0}^{2} z_{i}(t) E\left[U_{i}(u)\right]=z_{0}, \\
& E[y(t, u)]=\sum_{i=0}^{2} y_{i}(t) E\left[U_{i}(u)\right]=y_{0}, \\
& E[q(t, u)]=\sum_{i=0}^{2} q_{i}(t) E\left[U_{i}(u)\right]=q_{0} .
\end{aligned}
$$

The responses $x(t)$ and $y(t)$ of the deterministic equation (6) can be directly obtained by the Runge-Kutta method. The initial condition for the equivalent deterministic system (equation (10)) is

$$
\left\{\begin{array}{l}
x(0)=[0.18,0,0]^{T} \\
z(0)=[0,0,0]^{T} \\
y(0)=[0.18,0,0]^{T} \\
q(0)=[0,0,0]^{T} .
\end{array}\right.
$$

\section{Hopf Bifurcation Analysis of Equivalent Deterministic Magnetic Bearing System}

3.1. Mathematical Analysis. This section studies the Hopf bifurcation of the equivalent magnetic bearing (equation (10)) through theoretical analysis. We consider the situation when the magnetic force $f$ disappears. Obviously, the equivalent magnetic bearing deterministic system has a unique equilibrium point $x_{i 0}$, the Jacobian matrix of equation (10) is

$$
J=\left[\begin{array}{ccccccc}
0 & 1 & 0 & 0 & \cdots & 0 & 0 \\
-\omega^{2} & -2 \bar{\mu} & 0 & 0 & \cdots & 0 & 0 \\
0 & 0 & 0 & 1 & \cdots & 0 & 0 \\
0 & 0 & -\omega^{2} & -2 \bar{\mu} & \cdots & 0 & 0 \\
\cdots & \cdots & \cdots & \cdots & \ddots & \cdots & \cdots \\
0 & 0 & 0 & 0 & \cdots & 0 & 1 \\
0 & 0 & 0 & 0 & \cdots & -\omega^{2} & -2 \bar{\mu}
\end{array}\right] .
$$

The characteristic equation of the matrix (15) is

$$
\begin{aligned}
F(\lambda)= & a_{0} \lambda^{12}+a_{1} \lambda^{11}+a_{2} \lambda^{10}+a_{3} \lambda^{9}+a_{4} \lambda^{8}+a_{5} \lambda^{7}+a_{6} \lambda^{6} \\
& +a_{7} \lambda^{5}+a_{8} \lambda^{4}+a_{9} \lambda^{3}+a_{10} \lambda^{2}+a_{11} \lambda^{1}+a_{12} \\
= & 0
\end{aligned}
$$

where $a_{i}(i=1,2, \ldots, 12)$ can be calculated by MATLAB. According to the deterministic nonlinear dynamical system bifurcation theory, we assume that the bifurcation parameter is $v$; the conditions under which Hopf bifurcation occurs in the system are

(1) The Jacobi matrix (15) has a pair of conjugated eigenvalues

(2) When $v=v_{c}, \alpha\left(v_{c}\right)=0, \beta\left(v_{c}\right)>0$, and $\alpha^{\prime}\left(v_{v}\right) \neq 0$

(3) The rest eigenvalues of Jacobi matrix (15) have nonzero real parts

Since the order of the equivalence equations is high, it is difficult to find the parameter value that satisfies the above conditions directly. Therefore, the following lemma is proposed for obtaining the parameter value.

Lemma 1. Let $F$ be the characteristic equation of the system at the equilibrium point, $\Delta_{n}$ is the n-dimensional RouthHurwitz determinant, and $a_{i}$ is the coefficient of the characteristic equation. If a bifurcation parameter $v_{c}$ satisfies

(1) $\Delta_{n-1}\left(v_{c}\right)=0, \Delta_{n-2}\left(v_{c}\right) \neq 0, \Delta_{n-3}\left(v_{c}\right) \neq 0$

(2) $a_{i}\left(v_{c}\right)>0(i=0, \ldots, n)$

(3) $\Delta_{n-1}^{\prime}\left(v_{c}\right) \neq 0$

Then, the first two of Hopf existence conditions are established.

According to [6], we substitute $\bar{\mu}=0.019, \omega=1.0085$ into $\Delta_{11}=0$ and get the value of the bifurcation parameter $\delta$. Only when $\delta=0.025$, there exists $\Delta_{10}(\delta) \neq 0, \Delta_{9}(\delta) \neq 0$, $\Delta_{11}^{\prime}(\delta) \neq 0, \quad a_{i}(\delta)>0(i=0, \ldots, 12)$, and the first two 
conditions of the Hopf bifurcation are established. Also at this time, the eigenvalues of the matrix are $\lambda_{1,2}=-0.019 \mp 1.0083 i$, $\lambda_{3,4}=-0.0367 \mp 1.0078 i$, and $\lambda_{5,6}=-0.0013 \mp 1.0085 i$. Obviously, the matrix (15) has conjugate eigenvalues and the real parts of the remaining eigenvalues are negative. Therefore, the bearing system with uncertain parameter appears Hopf bifurcation near the zero when the system's bifurcation parameter $\delta=0.025$.

3.2. Numerical Simulation. To perform the numerical simulations, the values for the system parameters are chosen as follows: $\omega=1.0085, \alpha_{1}=-0.9262, \alpha_{2}=1.8591, \alpha_{3}=$ $-0.0113, \quad \alpha_{4}=-0.0162, \alpha_{5}=2.9289 \times 10^{-5}, \quad \alpha_{6}=1.7071 \times$ $10^{-4}$, and $\alpha_{7}=-0.0182$. Considering the equivalent deterministic magnetic bearing system with $f=0$, from the above analysis, the critical value for generating Hopf bifurcation is $\delta=0.025$. When $\delta<0.025$, the horizontal and vertical responses of the magnetic bearing system are as shown in Figure 3, and the responses in both directions are the same. The SRM and EMR phase trajectory gradually converge to the equilibrium point with time. The corresponding time history diagram shows that the response solution fluctuates more slowly and tends to the line gradually. In other words, if the initial value is given, the magnetic force disappears suddenly and the motion of the magnetic bearing system tends to stop eventually. It can be seen from Figure 3 that the horizontal and vertical magnetic bearing movements are the same when $f=0$, so we only consider the horizontal direction in the subsequent research. As $\delta>0.025$, a limit cycle is generated at the equilibrium point, as shown in Figure 4. The phase trajectory of the SRM and EMR gradually converge to a closed curve near the equilibrium point, that is, the equivalent deterministic magnetic bearing system creates a limit cycle. The amplitude of the EMR no longer changes which can be seen from the time history diagram. The trajectory at this moment indicates that the unstable oscillation of the corresponding magnetic bearing system occurs.

\section{Hopf Bifurcation Control}

In practical applications, complex magnetic bearing systems are generally considered to be deterministic. Routine maintenance and monitoring are performed as deterministic; in fact, almost all actual models are affected by random factors. If the effects of randomness are not eliminated, the magnetic bearing system is likely to have many unexpected disruptive behaviors. In order to overcome the impact of randomness, we introduce a hybrid feedback controller to achieve the goal.

4.1. Linear Feedback Control Method. Linear feedback control method is a kind of common method in previous papers. We use this method on the stochastic parameter model. Let controllers be $C_{10}=k_{2} z$ and $C_{11}=k_{2} q$. Put the controller on the bearing's model, and the model becomes equation (17). Then, we can get equation (18) by the orthogonal polynomial approximation theory as used before:

$$
\begin{aligned}
\dot{x}= & z, \\
\dot{z}= & \alpha_{4} y^{2} z-2(\bar{\mu}+\delta u) z+\alpha_{3} x^{2} z+\alpha_{6} x z^{2}+\alpha_{1} x^{3} \\
& +\left(\alpha_{2} y^{2}+\alpha_{5} q^{2}+\alpha_{7} y q-\omega^{2}\right) x+k_{2} z, \\
\dot{y}= & q, \\
\dot{q}= & \alpha_{4} x^{2} q-2(\bar{\mu}+\delta u) q+\alpha_{3} y^{2} q+\alpha_{6} y q^{2}+\alpha_{1} y^{3} \\
& +\left(\alpha_{2} x^{2}+\alpha_{5} z^{2}+\alpha_{7} x z-\omega^{2}\right) y+k_{2} q,
\end{aligned}
$$

$$
\begin{aligned}
\frac{\mathrm{d}}{\mathrm{d} t} x_{0}(t)= & z_{0}(t) \\
\frac{\mathrm{d}}{\mathrm{d} t} z_{0}(t)= & \alpha_{4} S_{0}(t)-2 \bar{\mu} z_{0}(t)-\delta z_{1}(t)+\alpha_{3} A_{0}(t) \\
& +\alpha_{6} B_{0}(t)+\alpha_{1} X_{0}(t)+\alpha_{2} C_{0}(t)+\alpha_{5} D_{0}(t) \\
& +\alpha_{7} E_{0}(t)-\omega^{2} x_{0}(t)+k_{2} z_{0},
\end{aligned}
$$$$
\frac{\mathrm{d}}{\mathrm{d} t} y_{0}(t)=q_{0}(t)
$$$$
\frac{\mathrm{d}}{\mathrm{d} t} q_{0}(t)=\alpha_{4} F_{0}(t)-2 \bar{\mu} q_{0}(t)-\delta q_{1}(t)+\alpha_{3} G_{0}(t)
$$$$
+\alpha_{6} H_{0}(t)+\alpha_{1} Y_{0}(t)+\alpha_{2} I_{0}(t)+\alpha_{5} J_{0}(t)
$$$$
+\alpha_{7} K_{0}(t)-\omega^{2} y_{0}(t)+k_{2} q_{0} \text {, }
$$$$
\frac{\mathrm{d}}{\mathrm{d} t} x_{1}(t)=z_{1}(t)
$$$$
\frac{\mathrm{d}}{\mathrm{d} t} z_{1}(t)=\alpha_{4} S_{1}(t)-2 \bar{\mu} z_{1}(t)-\delta\left(z_{0}(t)+z_{2}(t)\right)
$$$$
+\alpha_{3} A_{1}(t)+\alpha_{6} B_{1}(t)+\alpha_{1} X_{1}(t)+\alpha_{2} C_{1}(t)
$$$$
+\alpha_{5} D_{1}(t)+\alpha_{7} E_{1}(t)-\omega^{2} x_{1}(t)+k_{2} z_{1} \text {, }
$$

$$
\begin{aligned}
\frac{\mathrm{d}}{\mathrm{d} t} y_{1}(t)= & q_{1}(t) \\
\frac{\mathrm{d}}{\mathrm{d} t} q_{1}(t)= & \alpha_{4} F_{1}(t)-2 \bar{\mu} q_{1}(t)-\delta\left[q_{0}(t)+q_{2}(t)\right] \\
& +\alpha_{3} G_{1}(t)+\alpha_{6} H_{1}(t)+\alpha_{1} Y_{1}(t)+\alpha_{2} I_{1}(t) \\
& +\alpha_{5} J_{1}(t)+\alpha_{7} K_{1}(t)-\omega^{2} y_{1}(t)+k_{2} q_{1}, \\
\frac{\mathrm{d}}{\mathrm{d} t} x_{2}(t)= & z_{2}(t) \\
\frac{\mathrm{d}}{\mathrm{d} t} z_{2}(t)= & \alpha_{4} S_{2}(t)-2 \bar{\mu} z_{2}(t)-\delta z_{1}(t)+\alpha_{3} A_{2}(t) \\
& +\alpha_{6} B_{2}(t)+\alpha_{1} X_{2}(t)+\alpha_{2} C_{2}(t)+\alpha_{5} D_{2}(t) \\
& +\alpha_{7} E_{2}(t)-\omega^{2} x_{2}(t)+k_{2} z_{2}, \\
\frac{\mathrm{d}}{\mathrm{d} t} y_{2}(t)= & q_{2}(t), \\
\frac{\mathrm{d}}{\mathrm{d} t} q_{2}(t)= & \alpha_{4} F_{2}(t)-2 \bar{\mu} q_{2}(t)-\delta q_{1}(t)+\alpha_{3} G_{2}(t) \\
& +\alpha_{6} H_{2}(t)+\alpha_{1} Y_{2}(t)+\alpha_{2} I_{2}(t)+\alpha_{5} J_{2}(t) \\
& +\alpha_{7} K_{2}(t)-\omega^{2} y_{2}(t)+k_{2} q_{2} .
\end{aligned}
$$




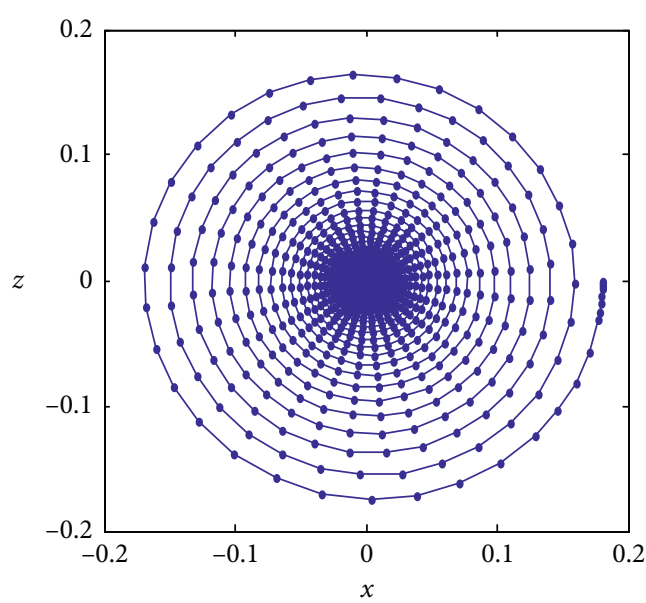

- SRM

- EMR

(a)

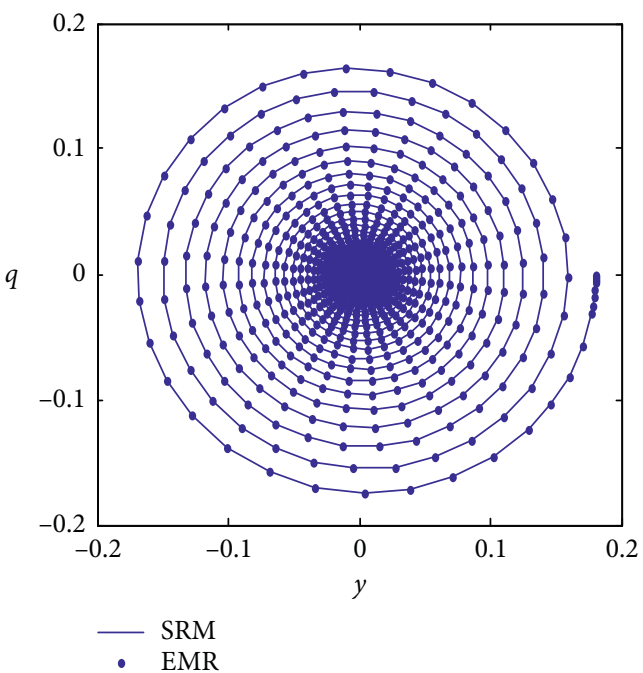

(c)

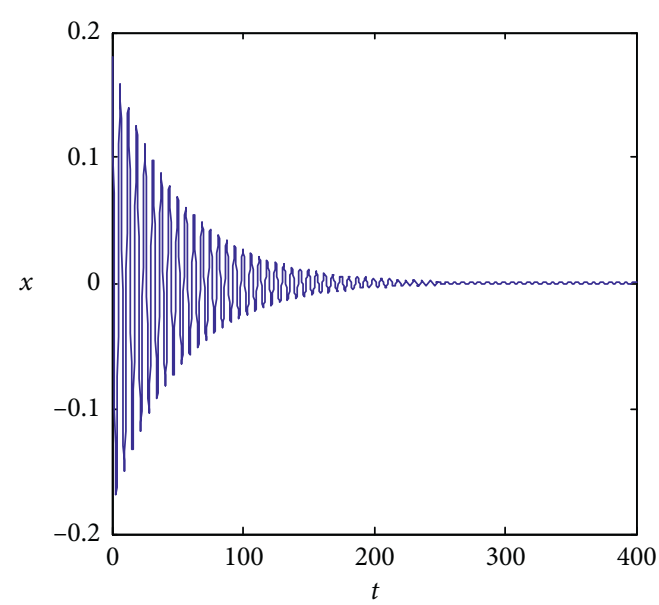

(b)

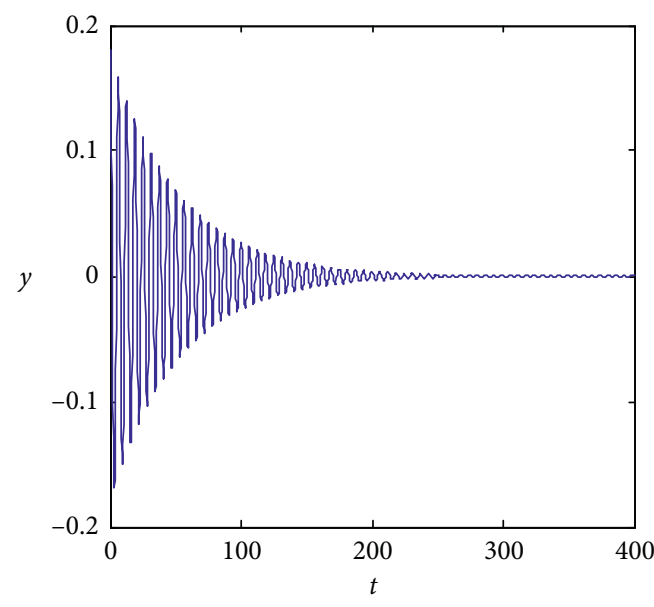

(d)

FIGURE 3: Uncertain magnetic bearing system response and time history diagram when $f=0, \delta<0.025$. (a) The response of horizontal direction. (b) Time history diagram of horizontal direction. (c) The response of vertical direction. (d) Time history diagram of vertical direction.

According to equation (18), the phase orbit diagram and time history diagram when $k_{2}=0.01$ are shown in Figure 5. Through comparing Figure 4(a) with Figure 5(a), we can find that the amplitude of the limit cycle decreases. That is to say, the linear feedback control method is effective.

4.2. Nonlinear Stochastic Feedback Control. A nonlinear stochastic feedback controller for the Hopf bifurcation of the uncertain magnetic bearing model is proposed firstly in [23]. Let the feedback controller be $C_{20}=k_{1}\left(\delta_{1} u^{\prime}-1\right) x^{2} z$ and $C_{21}=k_{1}\left(\delta_{1} u^{\prime}-1\right) y^{2} q$, where $k_{1}$ is the feedback strength, when $\delta_{1}$ is the random strength of the controller, and $u^{\prime}$ is a random variable that is independent distributed with the variables in the stochastic system. A controlled system loaded with a nonlinear stochastic controller can be written as

$$
\begin{aligned}
\dot{x}= & z, \\
\dot{z}= & \alpha_{4} y^{2} z-2(\bar{\mu}+\delta u) z+\alpha_{3} x^{2} z+\alpha_{6} x z^{2}+\alpha_{1} x^{3} \\
& +\left(\alpha_{2} y^{2}+\alpha_{5} q^{2}+\alpha_{7} y q-\omega^{2}\right) x+k_{1}\left(\delta_{1} u^{\prime}-1\right) x^{2} z, \\
\dot{y}= & q, \\
\dot{q}= & \alpha_{4} x^{2} q-2(\bar{\mu}+\delta u) q+\alpha_{3} y^{2} q+\alpha_{6} y q^{2}+\alpha_{1} y^{3} \\
& +\left(\alpha_{2} x^{2}+\alpha_{5} z^{2}+\alpha_{7} x z-\omega^{2}\right) y+k_{1}\left(\delta_{1} u^{\prime}-1\right) y^{2} q .
\end{aligned}
$$

A controlled system, equation (19), above can be reduced 


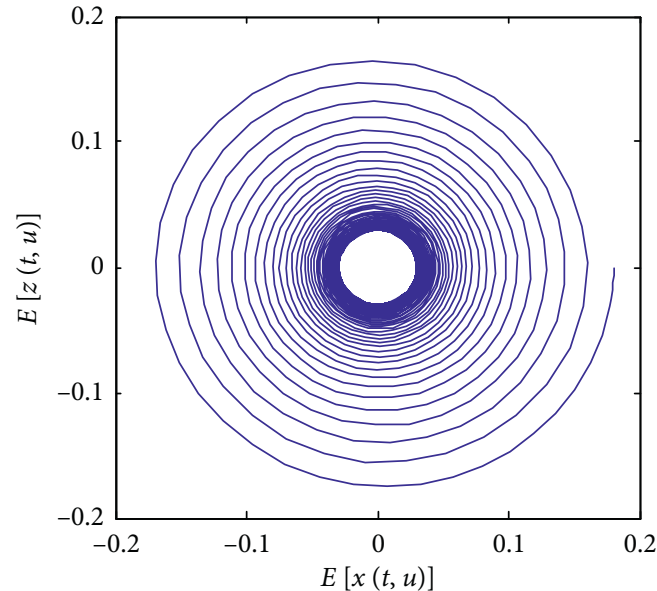

(a)

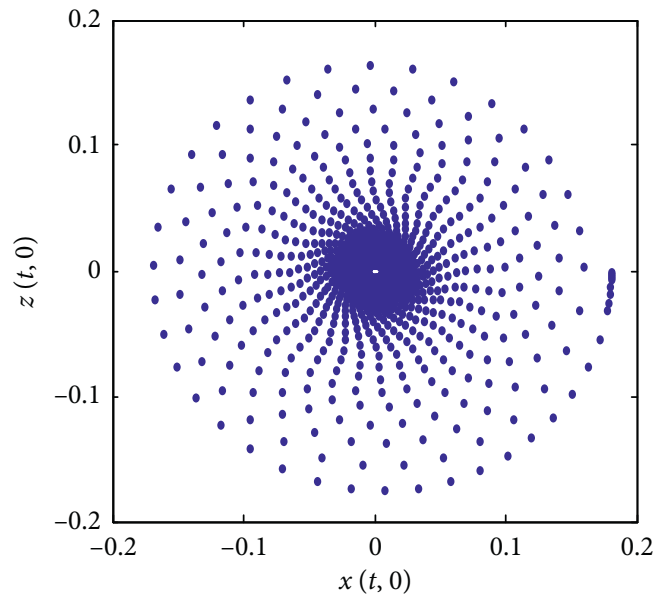

(c)

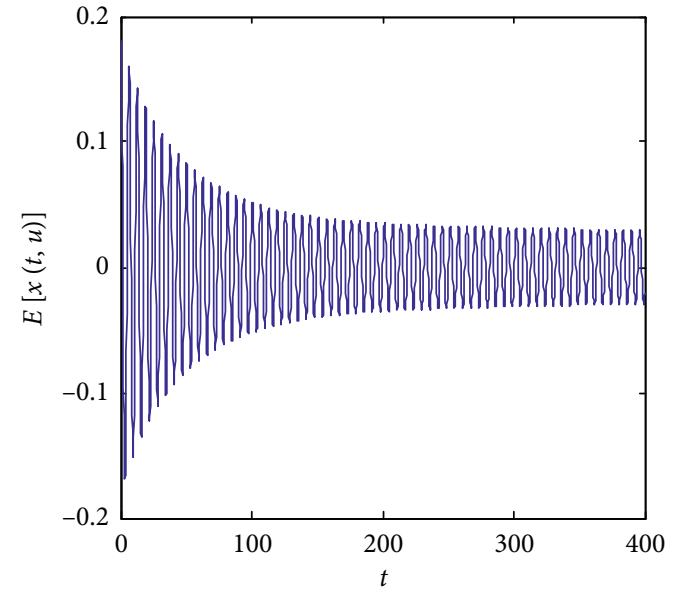

(b)

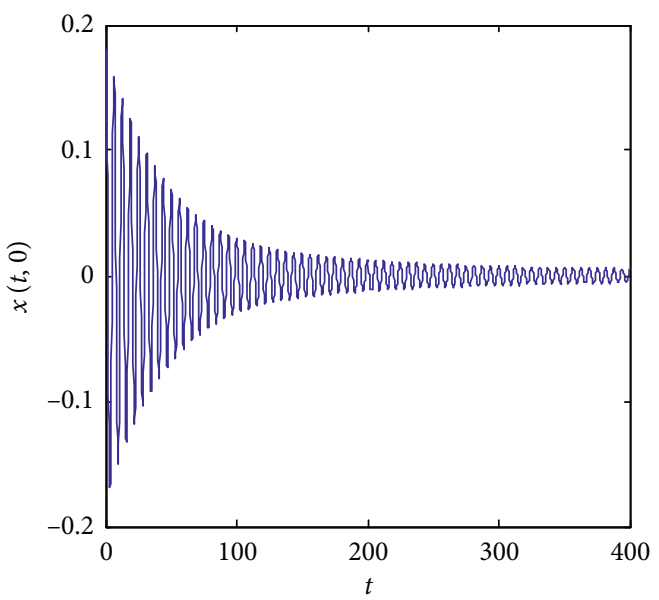

(d)

FIGURE 4: Uncertain magnetic bearing system response and time history diagram when $f=0, \delta>0.025$. (a) The EMR of horizontal direction. (b) Time history diagram of horizontal direction. (c) The SRM of horizontal direction. (d) Time history diagram of horizontal direction.

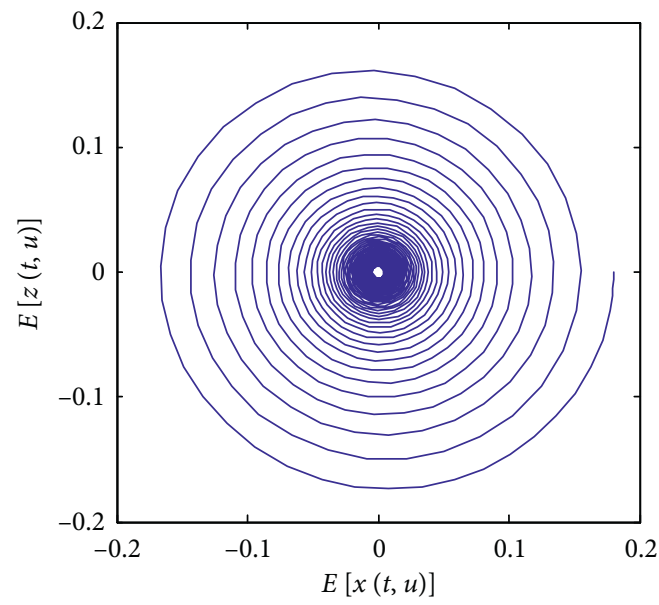

(a)

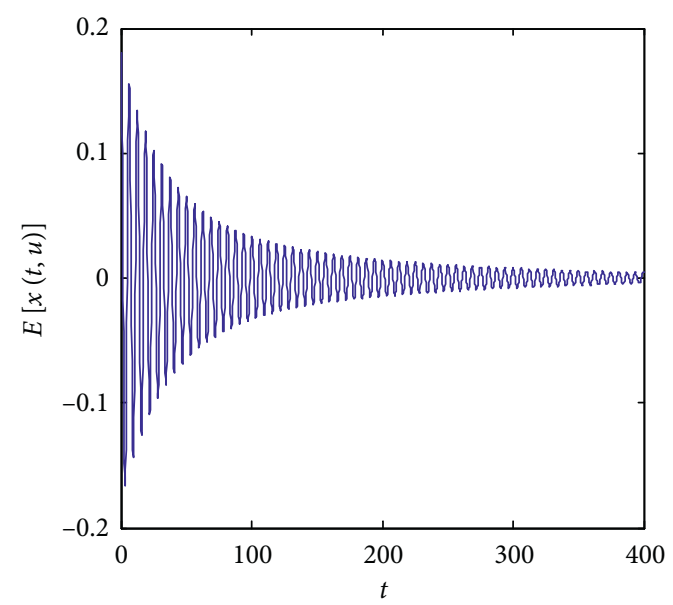

(b)

FIgURE 5: Response diagram and time history diagram of uncertain magnetic bearing system controlled by linear feedback. 


$$
\begin{aligned}
\frac{\mathrm{d}}{\mathrm{d} t} x_{0}(t)= & z_{0}(t), \\
\frac{\mathrm{d}}{\mathrm{d} t} z_{0}(t)= & \alpha_{4} S_{0}(t)-2 \bar{\mu} z_{0}(t)-\delta z_{1}(t)+\alpha_{3} A_{0}(t) \\
& +\frac{\delta_{1}}{2} k_{1} A_{1}(t)-k_{1} A_{0}(t)+\alpha_{6} B_{0}(t)+\alpha_{1} X_{0}(t) \\
& +\alpha_{2} C_{0}(t)+\alpha_{5} D_{0}(t)+\alpha_{7} E_{0}(t)-\omega^{2} x_{0}(t), \\
\frac{\mathrm{d}}{\mathrm{d} t} y_{0}(t)= & q_{0}(t), \\
\frac{\mathrm{d}}{\mathrm{d} t} q_{0}(t)= & \alpha_{4} F_{0}(t)-2 \bar{\mu} q_{0}(t)-\delta q_{1}(t)+\alpha_{3} G_{0}(t) \\
& +\frac{\delta_{1}}{2} k_{1} G_{1}(t)-k_{1} G_{0}(t)+\alpha_{6} H_{0}(t)+\alpha_{1} Y_{0}(t) \\
& +\alpha_{2} I_{0}(t)+\alpha_{5} J_{0}(t)+\alpha_{7} K_{0}(t)-\omega^{2} y_{0}(t),
\end{aligned}
$$

$$
\begin{aligned}
\frac{\mathrm{d}}{\mathrm{d} t} x_{1}(t)= & z_{1}(t), \\
\frac{\mathrm{d}}{\mathrm{d} t} z_{1}(t)= & \alpha_{4} S_{1}(t)-2 \bar{\mu} z_{1}(t)-\delta\left(z_{0}(t)+z_{2}(t)\right)+\alpha_{3} A_{1}(t) \\
& +\frac{\delta_{1}}{2} k_{1}\left(A_{0}(t)+A_{2}(t)\right)-k_{1} A_{1}(t)+\alpha_{6} B_{1}(t) \\
& +\alpha_{1} X_{1}(t)+\alpha_{2} C_{1}(t)+\alpha_{5} D_{1}(t)+\alpha_{7} E_{1}(t) \\
& -\omega^{2} x_{1}(t),
\end{aligned}
$$$$
\frac{\mathrm{d}}{\mathrm{d} t} y_{1}(t)=q_{1}(t)
$$$$
\frac{\mathrm{d}}{\mathrm{d} t} q_{1}(t)=\alpha_{4} F_{1}(t)-2 \bar{\mu} q_{1}(t)-\delta\left[q_{0}(t)+q_{2}(t)\right]+\alpha_{3} G_{1}(t)
$$$$
+\frac{\delta_{1}}{2} k_{1}\left(G_{0}(t)+G_{2}(t)\right)-k_{1} G_{1}(t)+\alpha_{6} H_{1}(t)
$$$$
+\alpha_{1} Y_{1}(t)+\alpha_{2} I_{1}(t)+\alpha_{5} J_{1}(t)+\alpha_{7} K_{1}(t)
$$$$
-\omega^{2} y_{1}(t)
$$$$
\frac{\mathrm{d}}{\mathrm{d} t} x_{2}(t)=z_{2}(t)
$$$$
\frac{\mathrm{d}}{\mathrm{d} t} z_{2}(t)=\alpha_{4} S_{2}(t)-2 \bar{\mu} z_{2}(t)-\delta z_{1}(t)+\alpha_{3} A_{2}(t)
$$$$
+\frac{\delta_{1}}{2} k_{1} A_{1}(t)-k_{1} A_{2}(t)+\alpha_{6} B_{2}(t)+\alpha_{1} X_{2}(t)
$$$$
+\alpha_{2} C_{2}(t)+\alpha_{5} D_{2}(t)+\alpha_{7} E_{2}(t)-\omega^{2} x_{2}(t),
$$

$$
\begin{aligned}
\frac{\mathrm{d}}{\mathrm{d} t} y_{2}(t)= & q_{2}(t) \\
\frac{\mathrm{d}}{\mathrm{d} t} q_{2}(t)= & \alpha_{4} F_{2}(t)-2 \bar{\mu} q_{2}(t)-\delta q_{1}(t)+\alpha_{3} G_{2}(t) \\
& +\frac{\delta_{1}}{2} k_{1} G_{1}(t)-k_{1} G_{2}(t)+\alpha_{6} H_{2}(t)+\alpha_{1} Y_{2}(t) \\
& +\alpha_{2} I_{2}(t)+\alpha_{5} J_{2}(t)+\alpha_{7} K_{2}(t)-\omega^{2} y_{2}(t) .
\end{aligned}
$$

It can be seen from the above equations that when the strength of the controller takes $N=5$, and the strength value of controller is 3 . Considering $\delta_{1}=3$, the controlled magnetic bearing limit circle is shown in Figure 6(a). The time history diagram of the controlled uncertain magnetic bearing system is shown in Figure 6(b). It can be seen from Figure 6 that the nonlinear feedback control can also reduce the amplitude of the limit cycle, and the results are the same as that of the linear feedback controller nearly.

4.3. Hybrid Feedback Control Method. In this part, we carry out a new method which is a kind of hybrid method. This method is a linear feedback controller combined with nonlinear stochastic feedback controller. Of course, we want to know if this method is more effective than either of the single method. We design a hybrid feedback controller is $C_{30}=k_{2} z+k_{1}\left(\delta_{1} u^{\prime}-1\right) x^{2} z$ and $C_{31}=k_{2} q+k_{1}\left(\delta_{1} u^{\prime}-1\right)$ $y^{2} q$. The values of the coefficients of hybrid feedback controller are the same as in Sections 4.1 and 4.2. Then, the stochastic magnetic bearing controlled by the hybrid feedback method can be written as

$$
\begin{aligned}
\dot{x}= & z, \\
\dot{z}= & \alpha_{4} y^{2} z-2(\bar{\mu}+\delta u) z+\alpha_{3} x^{2} z+\alpha_{6} x z^{2}+\alpha_{1} x^{3} \\
& +\left(\alpha_{2} y^{2}+\alpha_{5} q^{2}+\alpha_{7} y q-\omega^{2}\right) x+k_{2} z+k_{1}\left(\delta_{1} u^{\prime}-1\right) x^{2} z, \\
\dot{y}= & q, \\
\dot{q}= & \alpha_{4} x^{2} q-2(\bar{\mu}+\delta u) q+\alpha_{3} y^{2} q+\alpha_{6} y q^{2}+\alpha_{1} y^{3} \\
& +\left(\alpha_{2} x^{2}+\alpha_{5} z^{2}+\alpha_{7} x z-\omega^{2}\right) y+k_{2} q+k_{1}\left(\delta_{1} u^{\prime}-1\right) y^{2} q .
\end{aligned}
$$

The results of hybrid feedback controller are depicted based on equation (20) as shown in Figure 7. The RungeKutta method is utilized to explore the effectiveness of the new controller. It is observed from Figures 4(a), 4(b) and 7 that the hybrid controller method can reduce the amplitude of Hopf bifurcation. Furthermore, it is more useful to control the Hopf bifurcation than linear feedback controller and nonlinear stochastic feedback controller, and we can see this result by comparison of Figures 5 and 6 with Figure 7.

The time history diagrams of Figure 8 are depicted based on equations (18), (20), and (22), respectively. Figures 8(b) and $8(\mathrm{c})$ are two enlarged views of Figure $8(\mathrm{a})$. One can observe that a limit cycle is becoming smaller gradually, and when the hybrid controller is added to magnetic bearing, the Hopf bifurcation disappears nearly. Selecting different types of controllers, the result can be obtained. We plot the response and time history diagram of the magnetic bearing system to demonstrate the influence of three kinds of controllers. We compare the results of three controllers by the numerical method. Figure 8(b) demonstrates that the nonlinear stochastic feedback controller is better than the linear feedback controller in a certain period of time, while further numerical simulation in Figure 8(c) shows that two controllers have the same results for controlling Hopf bifurcation. But throughout this section, we have to point out that a hybrid controller is better than either of the single controller. The results can be verified explicitly by Figure 8 . Based on linear feedback controller and nonlinear stochastic feedback controller, the new hybrid controller presented by us is more effective: 


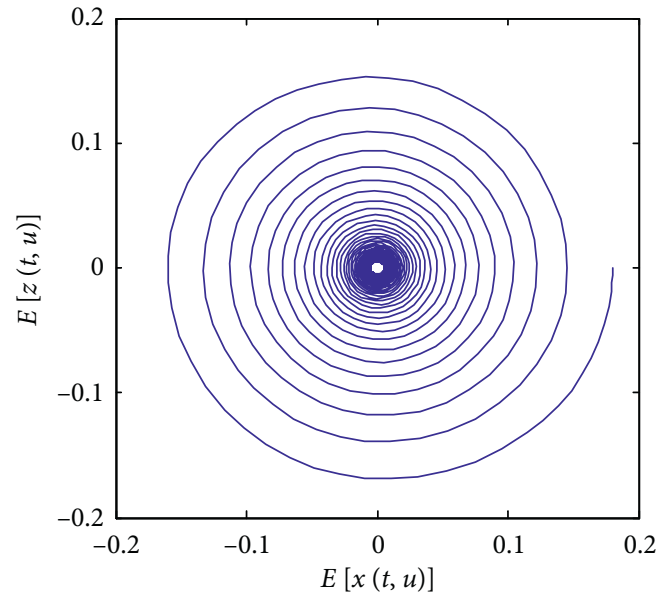

(a)

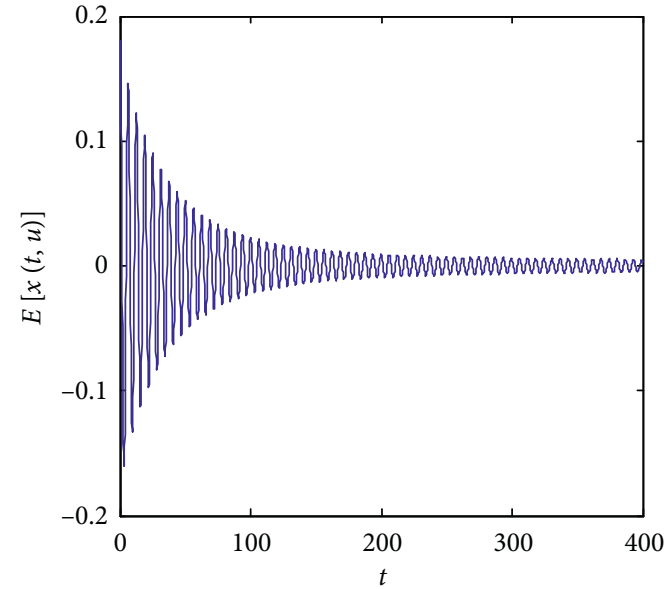

(b)

FIGURE 6: Response diagram and time history diagram of uncertain magnetic bearing system controlled by nonlinear stochastic feedback.

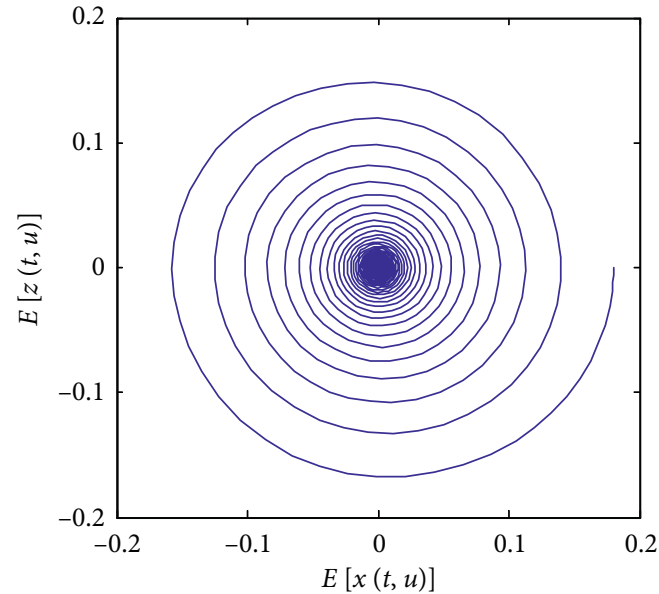

(a)

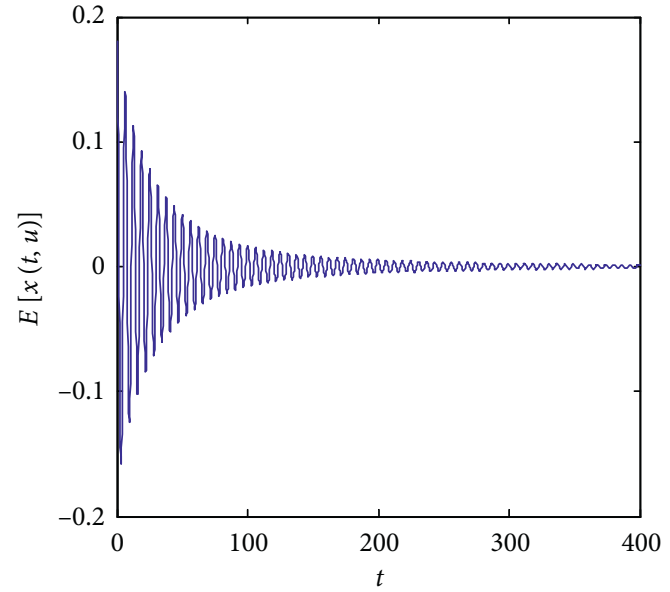

(b)

FiguRE 7: Response diagram and time history diagram of the uncertain magnetic bearing system controlled by a hybrid feedback method.

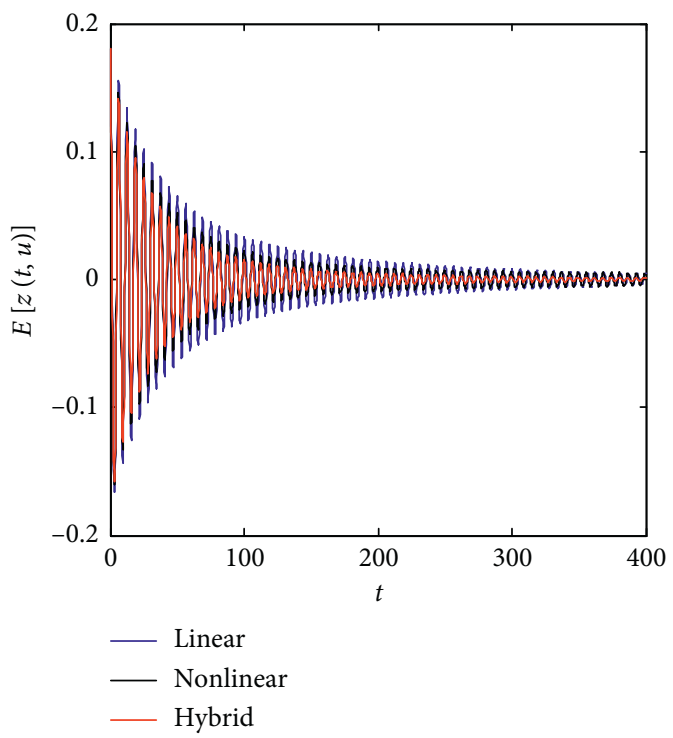

(a)

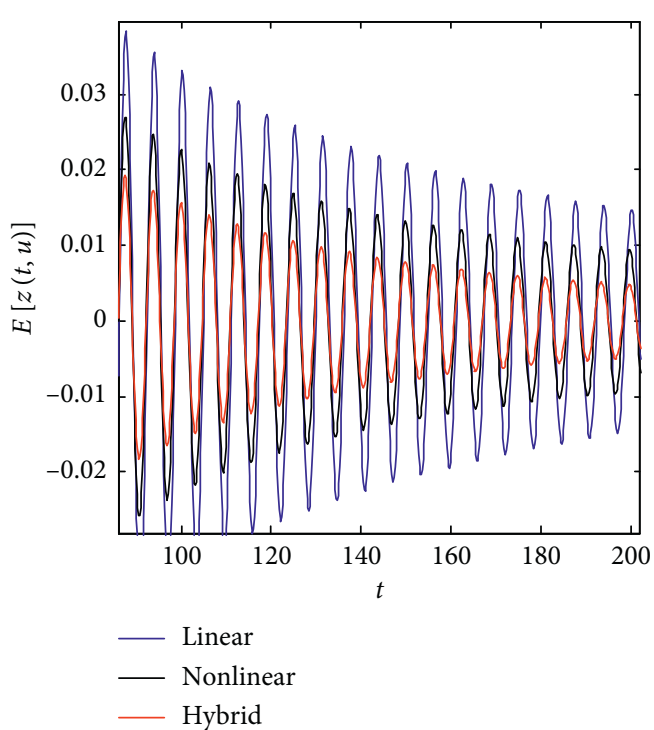

(b)

Figure 8: Continued. 


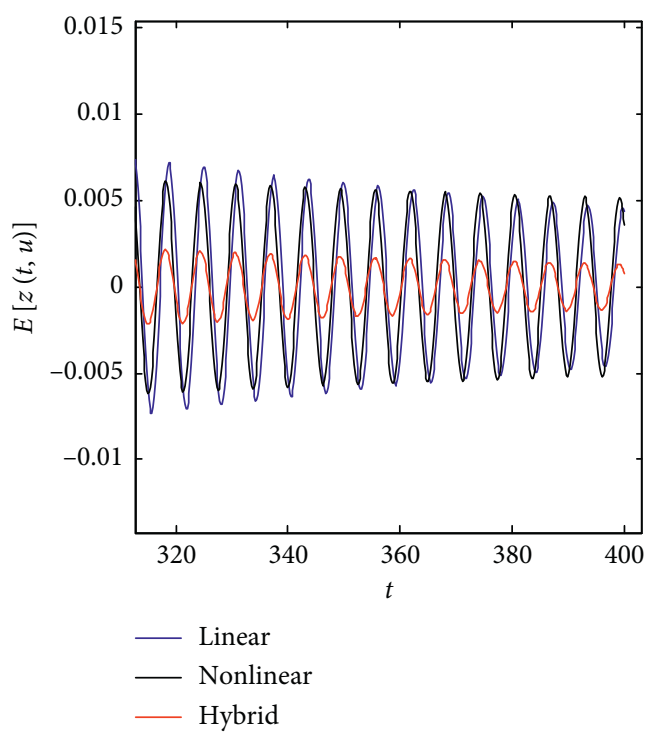

(c)

FIGURE 8: Time history diagrams of uncertain the magnetic bearing system controlled by three different methods. (b, c) Enlarged views of (a).

$$
\begin{aligned}
& \frac{\mathrm{d}}{\mathrm{d} t} x_{0}(t)=z_{0}(t) \\
& \frac{\mathrm{d}}{\mathrm{d} t} z_{0}(t)=\alpha_{4} S_{0}(t)-2 \bar{\mu} z_{0}(t)-\delta z_{1}(t)+\alpha_{3} A_{0}(t)+k_{2} z_{0} \\
& +\frac{\delta_{1}}{2} k_{1} A_{1}(t)-k_{1} A_{0}(t)+\alpha_{6} B_{0}(t)+\alpha_{1} X_{0}(t) \\
& +\alpha_{2} C_{0}(t)+\alpha_{5} D_{0}(t)+\alpha_{7} E_{0}(t)-\omega^{2} x_{0}(t) \\
& \frac{\mathrm{d}}{\mathrm{d} t} y_{0}(t)=q_{0}(t) \\
& \frac{\mathrm{d}}{\mathrm{d} t} q_{0}(t)=\alpha_{4} F_{0}(t)-2 \bar{\mu} q_{0}(t)-\delta q_{1}(t)+\alpha_{3} G_{0}(t)+k_{2} q_{0} \\
& +\frac{\delta_{1}}{2} k_{1} G_{1}(t)-k_{1} G_{0}(t)+\alpha_{6} H_{0}(t)+\alpha_{1} Y_{0}(t) \\
& +\alpha_{2} I_{0}(t)+\alpha_{5} J_{0}(t)+\alpha_{7} K_{0}(t)-\omega^{2} y_{0}(t) \\
& \frac{\mathrm{d}}{\mathrm{d} t} y_{1}(t)=q_{1}(t) \\
& \frac{\mathrm{d}}{\mathrm{d} t} q_{1}(t)=\alpha_{4} F_{1}(t)-2 \bar{\mu} q_{1}(t)-\delta\left[q_{0}(t)+q_{2}(t)\right] \\
& +\alpha_{3} G_{1}(t)+k_{2} q_{1}+\frac{\delta_{1}}{2} k_{1}\left(G_{0}(t)+G_{2}(t)\right) \\
& -k_{1} G_{1}(t)+\alpha_{6} H_{1}(t)+\alpha_{1} Y_{1}(t)+\alpha_{2} I_{1}(t) \\
& +\alpha_{5} J_{1}(t)+\alpha_{7} K_{1}(t)-\omega^{2} y_{1}(t) \\
& \frac{\mathrm{d}}{\mathrm{d} t} x_{2}(t)=z_{2}(t) \\
& \frac{\mathrm{d}}{\mathrm{d} t} z_{2}(t)=\alpha_{4} S_{2}(t)-2 \bar{\mu} z_{2}(t)-\delta z_{1}(t)+\alpha_{3} A_{2}(t)+k_{2} z_{2} \\
& +\frac{\delta_{1}}{2} k_{1} A_{1}(t)-k_{1} A_{2}(t)+\alpha_{6} B_{2}(t)+\alpha_{1} X_{2}(t) \\
& \frac{\mathrm{d}}{\mathrm{d} t} x_{1}(t)=z_{1}(t) \\
& \frac{\mathrm{d}}{\mathrm{d} t} z_{1}(t)=\alpha_{4} S_{1}(t)-2 \bar{\mu} z_{1}(t)-\delta\left(z_{0}(t)+z_{2}(t)\right) \\
& +\alpha_{3} A_{1}(t)+k_{2} z_{1}+\frac{\delta_{1}}{2} k_{1}\left(A_{0}(t)+A_{2}(t)\right) \\
& -k_{1} A_{1}(t)+\alpha_{6} B_{1}(t)+\alpha_{1} X_{1}(t)+\alpha_{2} C_{1}(t) \\
& +\alpha_{5} D_{1}(t)+\alpha_{7} E_{1}(t)-\omega^{2} x_{1}(t) \\
& +\alpha_{2} C_{2}(t)+\alpha_{5} D_{2}(t)+\alpha_{7} E_{2}(t)-\omega^{2} x_{2}(t) \\
& \frac{\mathrm{d}}{\mathrm{d} t} y_{2}(t)=q_{2}(t) \\
& \frac{\mathrm{d}}{\mathrm{d} t} q_{2}(t)=\alpha_{4} F_{2}(t)-2 \bar{\mu} q_{2}(t)-\delta q_{1}(t)+\alpha_{3} G_{2}(t)+k_{2} q_{2} \\
& +\frac{\delta_{1}}{2} k_{1} G_{1}(t)-k_{1} G_{2}(t)+\alpha_{6} H_{2}(t)+\alpha_{1} Y_{2}(t) \\
& +\alpha_{2} I_{2}(t)+\alpha_{5} J_{2}(t)+\alpha_{7} K_{2}(t)-\omega^{2} y_{2}(t)
\end{aligned}
$$




\section{Conclusions}

The nonlinear behavior of magnetic bearing has been investigated, and the uncertain parameter has been considered. It has been shown that the various permeability values of magnetic bearing can lead to Hopf bifurcation, indicating the importance of taking random parameter into account.

The dynamic system under uncertain parameter exhibits the Hopf bifurcation phenomena. The method of orthogonal polynomial approximation has been used to transform the system as an equivalent deterministic expansion system. The bifurcation behavior of deterministic system is studied for various permeability values. It has been shown that the motions for the $x$ and $y$ directions lose their stability via Hopf bifurcation. The results obtained by the mathematical theory method are in good agreement with numerical integration. Besides, a hybrid control strategy is presented which can stabilize the magnetic bearing more effective than traditional linear feedback controller and nonlinear stochastic feedback controller. An important advantage of this control method is not only regarding the combination of linear and nonlinear but also considering deterministic controller and stochastic controller.

The results obtained are expected to be useful in the know of magnetic bearing under uncertain parameters and designs of the controller to stabilize the magnetic bearing system.

\section{Nomenclature}

$\mu_{0}$ : Magnetic permeability under vacuum

$A$ : Effective cross-sectional area of one electromagnet

$N$ : The number of windings around the core

$I_{i}$ : Coil current

$\delta_{i}$ : Radial clearance between the stator and the rotor

$\varphi$ : Half angle of the radial electromagnetic circuit

$c_{0}:$ Steady-state air gap

$m$ : Mass

$e$ : Eccentricity of unbalance

$c$ : Damping coefficient

$\Omega$ : Rotor speed

$f_{i}$ : Electromagnetic force produced by every pair of electromagnets.

\section{Data Availability}

The data in my manuscript used to support the findings of this study have been deposited in the article named "NonLinear Oscillations of a Rotor in Active Magnetic Bearings" (doi:10.1006/jsvi.2000.3257).

\section{Conflicts of Interest}

The authors declare that there are no conflicts of interest regarding the publication of this paper.

\section{Acknowledgments}

This work was supported by the grants from the National Natural Science Foundation (no. 11772002), Ningxia Higher
Education First-Class Discipline Construction Funding Project (NXYLXK2017B09), Key Project of North Minzu University (no. 2017KJ14), and China Postdoctoral Fund Program (no. 2017M613251), Major Special Project of North Minzu University (no. ZDZX201902), "Light of the West" Talent Training Program of Chinese Academy of Sciences (no. XAB2016AW04), and the Research start-up fund of North Minzu University.

\section{Supplementary Materials}

The mathematical expression of capital letters in the paper formulas is shown in Supplementary Materials. (Supplementary Materials)

\section{References}

[1] J. C. Ji, C. H. Hansen, and A. C. Zander, "Nonlinear dynamics of magnetic bearing systems," Journal of Intelligent Material Systems and Structures, vol. 19, no. 12, pp. 1471-1491, 2008.

[2] W. Zhang and X. P. Zhan, "Periodic and chaotic motions of a rotor-active magnetic bearing with quadratic and cubic terms and time-varying stiffness," Nonlinear Dynamics, vol. 41, no. 4, pp. 331-359, 2005.

[3] J. C. Ji, "Stability and Hopf bifurcation of a magnetic bearing system with time delays," Journal of Sound and Vibration, vol. 259, no. 4, pp. 845-856, 2003.

[4] S. P. Harsha, K. Sandeep, and R. Prakash, "The effect of speed of balanced rotor on nonlinear vibrations associated with ball bearings," International Journal of Mechanical Sciences, vol. 45, no. 4, pp. 725-740, 2003.

[5] A. H. Nayfeh and B. Balachandran, "Experimental investigation of resonantly forced oscillations of a two-degreeof-freedom structure," International Journal of Non-linear Mechanics, vol. 25, no. 2-3, pp. 199-209, 1990.

[6] J. C. Ji and C. H. Hansen, "Non-linear oscillations of a rotor in active magnetic bearings," Journal of Sound and Vibration, vol. 240, no. 4, pp. 599-612, 2001.

[7] N. A. Saeed, M. Eissa, and W. A. El-Ganini, "Nonlinear oscillations of rotor active magnetic bearings system," Nonlinear Dynamics, vol. 74, no. 1-2, pp. 1-20, 2013.

[8] M.-J. Jang and C. O.-K. Chen, "Bifurcation analysis in flexible rotor supported by active magnetic bearing," International Journal of Bifurcation and Chaos, vol. 11, no. 8, pp. 2163-2178, 2001.

[9] J. I. Inayat-Hussain, "Geometric coupling effects on the bifurcations of a flexible rotor response in active magnetic bearings," Chaos, Solitons \& Fractals, vol. 41, no. 5, pp. 2664-2671, 2009.

[10] W. Zhang, M. H. Yao, and X. P. Zhan, "Multi-pulse chaotic motions of a rotor-active magnetic bearing system with timevarying stiffness," Chaos, Solitons \& Fractals, vol. 27, no. 1, pp. 175-186, 2006.

[11] M. Kamel and H. S. Bauomy, "Nonlinear study of a rotorAMB system under simultaneous primary-internal resonance," Applied Mathematical Modelling, vol. 34, no. 10, pp. 2763-2777, 2010.

[12] T. Inoue, Y. Ishida, and S. Murakami, “Theoretical analysis and experiments of the nonlinear vibration in a vertical rigid rotor supported by the magnetic bearing system," Journal of System Design and Dynamics, vol. 1, no. 2, pp. 295-306, 2007.

[13] S. Zhe, Z. Xiaoshen, F. Tianpeng et al., "Nonlinear dynamic characteristics analysis of active magnetic bearing system 
based on cell mapping method with a case study," Mechanical Systems and Signal Processing, vol. 117, pp. 116-137, 2019.

[14] M. Hutterer, G. Kalteis, and M. Schrödl, "Redundant unbalance compensation of an active magnetic bearing system," Mechanical Systems and Signal Processing, vol. 94, pp. 267278, 2017.

[15] X. Sun, B. Su, L. Chen, Z. Yang, X. Xu, and Z. Shi, "Precise control of a four degree-of-freedom permanent magnet biased active magnetic bearing system in a magnetically suspended direct-driven spindle using neural network inverse scheme," Mechanical Systems and Signal Processing, vol. 88, pp. 36-48, 2017.

[16] M. Kiani, H. Salarieh, A. Alasty, and S. M. Darbandi, "Hybrid control of a three-pole active magnetic bearing," Mechatronics, vol. 39, pp. 28-41, 2016.

[17] A. Shrivastava and A. R. Mohanty, "Estimation of single plane unbalance parameters of a rotor-bearing system using Kalman filtering based force estimation technique," Journal of Sound and Vibration, vol. 418, pp. 184-199, 2018.

[18] L. R. Da Oliveira, "Analysis of unbalances forces using methods of identification and finite elements," Mechanical Engineering and Automation, vol. 5, pp. 400-402, 2014.

[19] S. Zheng and R. Feng, "Feedforward compensation control of rotor imbalance for high-speed magnetically suspended centrifugal compressors using a novel adaptive notch filter," Journal of Sound and Vibration, vol. 366, pp. 1-14, 2016.

[20] X. Leng, G. Meng, T. Zhang, and T. Fang, "Bifurcation and chaos response of a cracked rotor with random disturbance," Journal of Sound and Vibration, vol. 299, no. 3, pp. 621-632, 2007.

[21] Z. Yan and L. H. Jia, "Seismic analysis of rotor system under stationary non-stationary random earthquake excitations," Chinese Journal of Computational Mechanics, vol. 19, no. 1, pp. 7-11, 2002.

[22] S. J. Ma, "Hopf bifurcation for a class of random van der pol systems," Journal of Physics, vol. 60, no. 1, pp. 44-50, 2011.

[23] Y. Xu, S. Ma, and H. Zhang, "Hopf bifurcation control for stochastic dynamical system with nonlinear random feedback method," Nonlinear Dynamics, vol. 65, no. 1-2, pp. 77-84, 2010. 


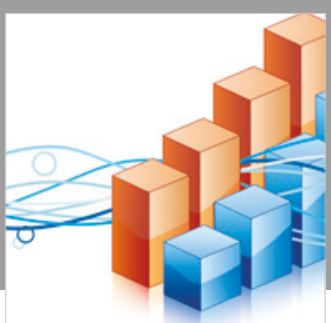

Advances in

Operations Research

\section{-n-m}
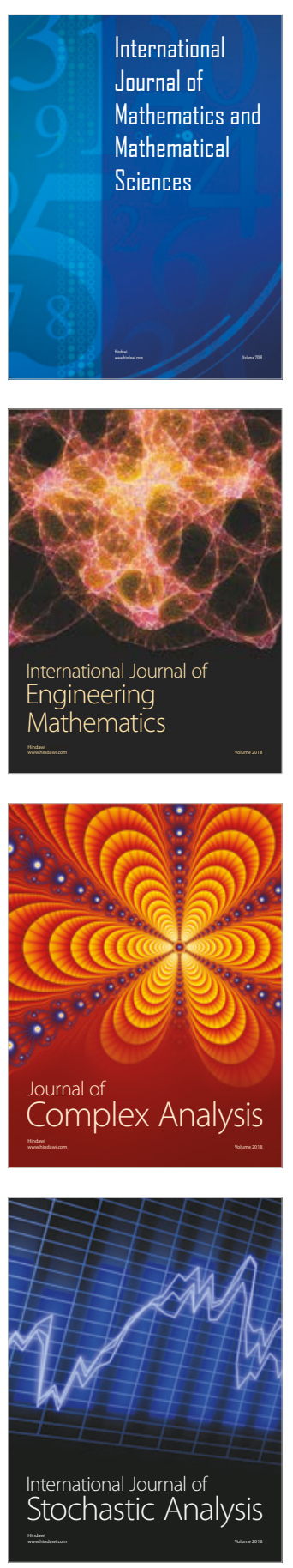
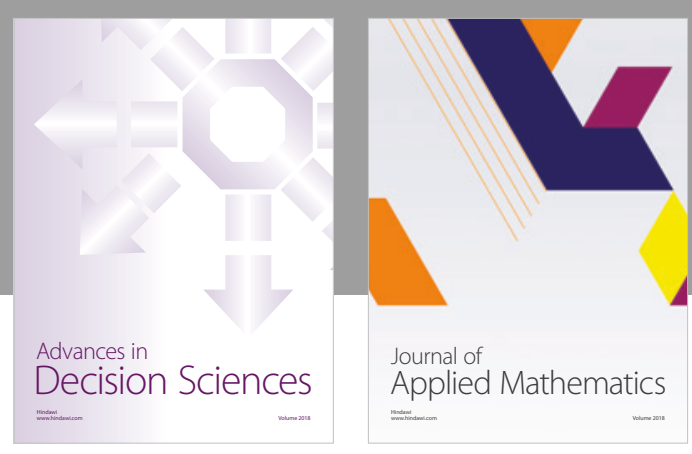

Journal of

Applied Mathematics
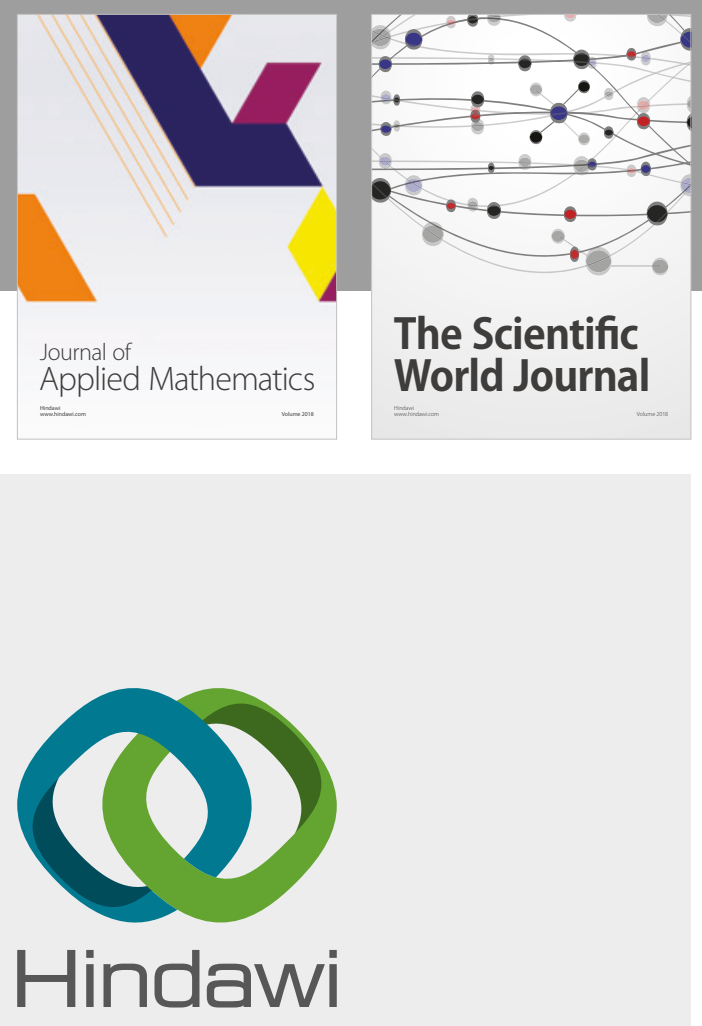

Submit your manuscripts at

www.hindawi.com

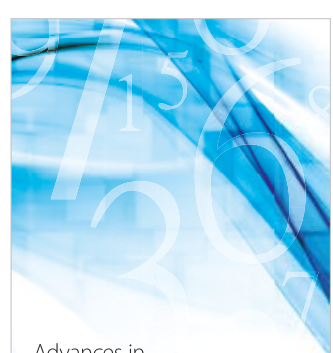

Advances in
Numerical Analysis
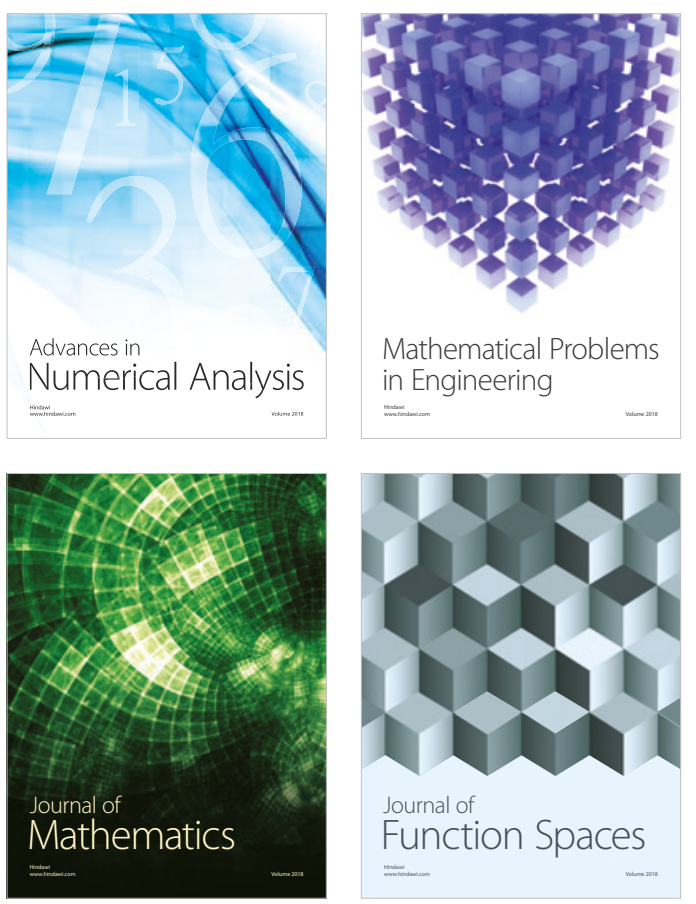

Mathematical Problems in Engineering

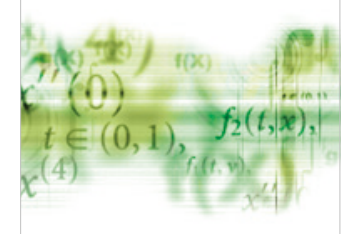

International Journal of

Differential Equations

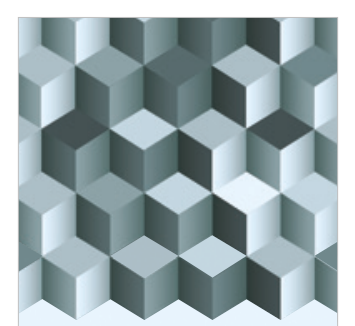

Journal of

Function Spaces
The Scientific

World Journal

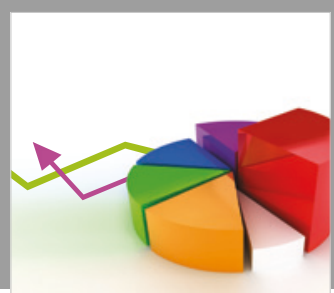

Journal of

Probability and Statistics
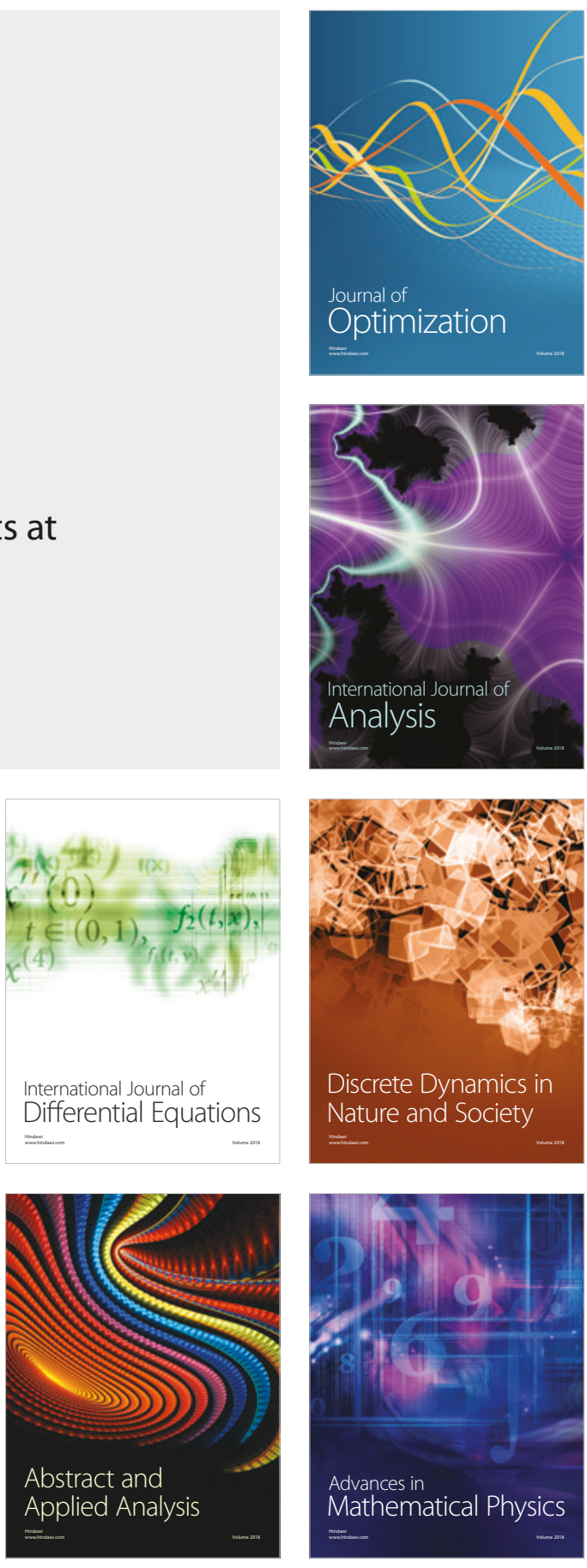